\title{
VISIÓN ACTUAL DEL SISTEMA JUDICIAL CHILENO
}

\author{
Urbano Marín Vallejo*
}

\section{Resumen:}

Este artículo explica la organización del sistema judicial del Poder Judicial Chileno, el cual actualmente atraviesa una etapa de transición, como consecuencia de profundas reformas que ha experimentado esta institución. Estos cambios han afectado la labor de los jueces y usuarios del Poder Judicial además de tener un alcance de orden social y cultural amplio y muy significativo.

Palabras clave: Sistema Judicial - Corte Suprema - Independencia Responsabilidad - Publicidad - Territorialidad - Inexcusabilidad Tribunales.

\begin{abstract}
:
This article explains the organization of the Chilean Judicial Power system, which is going through a transition stage as a consequence of the deep reform that this institution has experienced. These changes have affected the judge's work and the users of the service of this institution, as well as a deep and very significant social and cultural impact.
\end{abstract}

Key words: Judicial System - Supreme Court - Independence Responsibility - Publicity - Territoriality - Inexcusability - Tribunal.

Sumario:

1. Organización Institucional. 2. Bases Conceptuales. 3. Procedimientos Judiciales.

Ministro de la Corte Suprema de Justicia de Chile. 


\section{INTRODUCCIÓN}

Presentar una síntesis de un sistema judicial no sól o ofrece dificultades propias dela diversidad delos puntos devista quepueden considerarseal efecto. Ella se complica adicionalmente en el caso chileno, porque su justicia atraviesa una etapa de transición, como resultado de las profundas reformas que han experimentado tanto su organización como los procedimientos quesedesarrollan ante los tribunales. Estas innovaciones no sólo han afectado la situación de jueces y usuarios directos de la actividad judicial, sino que tienen alcances de orden social y cultural más amplios y significativos.

Por otra parte, desde hace largo tiempo, la comunidad nacional experimenta un acelerado fenómeno dejudicialización deasuntos públicos y privados, unido a una intensa mediatización de la justicia, aspectos que no dejan de estar presentes en el análisis, aún somero, del entorno judicial nacional.

En esta perspectiva, parece razonable limitarse a hacer, en primer lugar, una breve descripción de la estructura orgánica del sistema, para luego referirsea algunos de sus fundamentos conceptuales y terminar con una reseña de las reformas que seha puesto en práctica o que están en vías de implementarseen distintos sectores del sistema judicial chileno.

\section{ORGANIZACIÓN INSTITUCIONAL}

Como dice el profesor Mirjan Damaska, ${ }^{1}$ los sistemas judiciales reflejan la estructura orgánica del Estado. A sí debe ser, pues se refieren a una función pública esencial que para algunos lo justifica como entidad política y explica en parteel sometimiento del individuo a su autoridad.

La organización judicial deChilecorrespondea su condición deEstado unitario, ${ }^{2}$ quetiene un Gobierno Central dotado degran autoridad y facultades, con una descentralización en el plano administrativo, acentuada en el último tiempo, radicada en Intendencias y Gobiernos Regionales, Gobernaciones y en las Municipalidades, a cargo delas respectivas divisiones (Regiones, Provincias y Comunas) del territorio nacional.

Los tribunales configuran un Poder del Estado, con tal denominación desdela vigencia de la Constitución Política de 1925, pero su existencia y actividad se remontan al inicio de la época colonial, al crearse la Real Audiencia de la ciudad de Concepción en $1565 .^{3}$ 
Los tribunales queintegran el Poder Judicial son un archipiélago formado por 17 jurisdicciones a cargo deigual número de Cortes deA pelaciones, tribunales de segunda instancia delos que dependen cerca demedio millar detribunales de primer grado. Todos configuran un conjunto unitario con la forma de una pirámide, cuya cúspide es la Corte Suprema, a la que corresponde el gobierno central del Poder Judicial, pues, según el artículo 82으 de la Carta Política, tiene la superintendencia directiva, correccional y económica de los tribunales que lo forman.

Es el segundo Tribunal Supremo más antiguo en las A méricas, después de la CorteSuprema de Estados U nidos. Fuecreada en la Constitución de 1823, pero es sucesora de la aludida Real Audiencia y del Supremo Tribunal Judiciario establecido, a su vez, en 1811.

Como cabeza del Poder Judicial, configura el nivel máximo tanto en el ámbito jurisdiccional, como en el gobierno, dirección y admi nistración delajudicatura, sin perjuicio de las potestades que con autonomía jurisdiccional cumplen las Cortes de A pelaciones y todos los tribunales, en sus respectivos campos de competencia.

La CorteSuprema actúa como tribunal decasación, es decir, conocederecursos denulidad deforma y defondo respecto de ciertas sentencias de las Cortes de A pelaciones. A demás, es la segunda instancia en el terreno de las llamadas facultades conservadoras del os tribunales para asegurar el efectivo ejercicio de derechos garantizados por la Constitución Política (recursos de habeas corpus, de protección y otros). Hasta el 26 de febrero de 2006 conoció del recurso de inaplicabilidad de las leyes por inconstitucionalidad, que pasó al Tribunal Constitucional, ajeno al Poder Judicial. ${ }^{4}$

La CorteSuprema la forman 21 Ministros, queeligen entreellos a su Presidente, el que se mantiene dos años en su cargo. Funciona dividida en cuatro Salas especial izadas en asuntos civiles, penales, constitucionales y laborales y como Tribunal Pleno, especial menteen funciones degobierno judicial, admi nistración, disci plina y otras, con facul tad dedictar A utos A cordados para regular materias relativas al funcionamiento de los tribunales.

En la Justicia chilena existe una especialización sólo parcial en los tribunales, en primerainstanciay en la CorteSuprema. A partedelos tribunales degarantía y de juicio oral en el campo penal, están los tribunales de Familia creados a 
contar del 1 de octubre de 2005 , por la ley $\mathrm{N}^{\circ} 19.968$, de 30 de agosto de $2004 \mathrm{y}$ hay juzgados especiales del Trabajo y de Cobranza Previsional. Junto a ellos están los tribunales mixtos o dejurisdicción común que conocen detodo orden de materias o varias de ellas.

Las Cortes deA pelaciones no seencuentran especializadas y sus Sal as conocen indistintamente de toda clase de asuntos.

Los jueces chilenos son funcionarios profesional es permanentes en todos sus niveles, con dedicación exclusiva a sus cargos. Desarrollan su carrera como tales a través de concursos resueltos por los tribunales superiores. No hay participación ciudadana directa en los juicios ni en la designación de los jueces.

Colaboran en las funciones jurisdiccional es de la CorteSuprema y las Cortes de A pelaciones, reemplazando a los Ministros ausentes o impedidos de desempeñar sus cargos, un número variable de jueces adjuntos, llamados abogados integrantes, que se nombran por el poder ejecutivo mediante ternas propuestas por la CorteSuprema al Gobierno.

Hay tribunales especiales que no integran el Poder Judicial, pero respecto de los cuales la Corte Suprema ejerce al gún grado de supervisión: Ios Tribunales Militares y los Juzgados de Policía Local, quefuncionan en las Munici palidades y que conocen de infracciones penales menores (faltas) y otros asuntos. Otros tribunales están fuera de la autoridad de la Corte Suprema: Constitucional, Calificador General deElecciones y Calificadores Regionales.

Numerosos organismos administrativos, especial mentelos queejercen la función reguladora del Estado, tienen potestades sancionatorias sobre las personas y empresas quefiscal izan y sus resoluciones son objeto deredamaciones que conoce el Poder Judicial, a falta detribunal es contencioso-administrativos. Hay más de 140 procedimientos específicos de estetipo, aparte del recurso de protección de garantías constitucionales, que se suele utilizar para impugnar esas sanciones.

La gestión de los recursos humanos, financieros, materiales y tecnológicos que apoyan la función jurisdiccional de los tribunales, la hace la Corporación Administrativa del Poder Judicial, creada en 1990, para ejecutar esta función separadamente. Es dirigida por un Consejo que forman el Presidente y cuatro Ministros de la CorteSuprema y el Director del Organismo, pero su actividad está a cargo de un equipo de profesionales y técnicos que ha cumplido demodo 
eficiente su cometido, no sólo atendiendo las necesidades normales de los tribunales, sino haciendo frente sin fallas a las importantes reformas que han afectado al Poder Judicial, bajo la supervigilancia del Consejo y con el auxilio de auditorías externas y de una contral oría interna.

Finalmente, forma parte del Poder Judicial, como organismo autónomo, la A cademia Ju dicial, creada para formar postulantes a ingresar como jueces y a perfeccionarlos durante su carrera. En su Consejo Directivo está representada la Corte Suprema y ha cumplido un excelente desempeño en la preparación y capacitación delos recursos humanos para la implementación de las sucesivas reformas al sistema judicial.

\section{BASES CONCEPTUALES}

El sistema judicial chileno está construido sobre diversos cimientos teóricos que lo sustentan y que caracterizan su accionar. Entre otros, pueden indicarse los siguientes principios básicos:

\section{INDEPENDENCIA}

Esta característica es requisito necesario para quela acción de los tribunales se ejecute del modo recto eincontaminado de toda presión o influencia externa o interna.

Ella es más absoluta en lo estrictamente jurisdiccional y tiene reconocimiento constitucional explícito tanto positivo como negativo, pues la Carta Política chilena en su artículo 760 no sólo reserva a los tribunales la exclusividad del conocimiento decausales civiles y criminales, desu juzgamiento y dela ejecución delo resuelto, sino queprohíbedemodo terminanteal Presidentedela República y al Parlamento el ejercicio defunciones judiciales.

A su vez, el artícul o $4^{\circ}$ del Código Orgánico deTribunales veda al Poder Judicial mezclarse en las atribuciones de otros poderes públicos y desempeñar otras funciones quelas que determina ese cuerpo legal.

Los conflictos de competencia que pueden producirse entre tribunales y entre ellos y las autoridades políticas y administrativas se deciden a través de los mecanismos quecontemplan al efecto la Constitución y las leyes y queentregan el conocimiento de tales contiendas tanto al Senado como al Tribunal 


\section{Constitucional.}

La independencia judicial no tiene la misma extensión en todo el plano institucional. El financiamiento del Poder Judicial depende delos recursos que le asigne anualmente la Ley de Presupuestos de la Nación, que en materia de autorización de gastos es deiniciativa exclusiva del Ejecutivo. Hay un proyecto de ley en actual trámite parlamentario que concede mayor autonomía en el manejo de recursos presupuestarios al Poder Judicial.

La designación de los jueces se produce a través de una fórmula compuesta, especialmente en el caso de los Ministros de la Corte Suprema, quienes son nombradosatravés deuna quina queel mismo tribunal remiteal Presidentedela República, el que designaa uno desus integrantes y cuyo nombramiento debeser ratificado, a su vez, por el Senado. Cinco de los Ministros de la Corte Suprema deben designarse entre abogados externos al Poder Judicial que postulen al concurso quese convoqueal efecto y siguiendo el mismo procedimiento.

Todos los jueces son inamovibles en sus cargos hasta cumplir 75 años, pero el Presidente dela República puedesolicitar ala CorteSuprema su remoción por mal comportamiento. Los jueces de los tribunales superiores (CorteSuprema y Cortes deA pelaciones) pueden ser destituidos en un juicio político iniciado en la Cámara de Diputados y resuelto por Senado, por la causal de "notable abandono de sus deberes".

\section{RESPONSABILIDAD}

Esta noción es complementaria y correlativa de la anterior; la reconocetambién la Constitución Política (artículo 79우) y está desarrollada en el Código Orgánico deTribunales, queimponeobligaciones y prohi biciones a los jueces en la materia. Entre otros preceptos, el artículo 80 de la Carta Constitucional señala que el buen comportamiento de los jueces es condición para su permanencia en sus cargos y permite que sean removidos por la Corte Suprema a petición del Presidente dela República, de cualquiera persona o de oficio.

La responsabilidad de los jueces y demás funcionarios judiciales no es única y genérica, sino múltiple y de variada naturaleza. Se distinguen las responsabilidades política, penal, civil y disciplinaria que pueden afectarles y queaun cuando deriven del mismo hecho punible, conservan su identidad y se hacen efectivas por las vías específicas y con las sanciones que la normativa establece con ese objeto. 


\section{PUBLICIDAD}

Se trata de otra característica que complementa y refuerza las anteriores, en la medida que contribuye a la transparencia de las actuaciones judiciales.

El artículo $9^{\circ}$ del Código Orgánico de Tribunales señala que "los actos de los tribunales son públicos, sal vo las excepciones expresamenteestablecidas en la ley". Este era el caso, por ejemplo, del secreto del sumario criminal, es decir, la etapa del antiguo procedimiento penal relativa a la investigación del delito y la individualización de sus responsables y hoy rige para los acuerdos de los tribunales colegiados.

Después de la reforma dispuesta por la Ley $\mathrm{N}^{\circ} 20.050$, de 26 de agosto de 2005, el nuevo artículo $8^{\circ}$ de la Constitución Política expresa que "son públicos los actos y resoluciones de los órganos del Estado, así como sus fundamentos y los procedimientos queutilicen. Sin embargo, una ley dequórum calificado podrá establecer la reserva o secreto de aquéllos o éstos cuando la publicidad afectare al debido cumplimiento de las funciones de dichos órganos, los derechos de las personas, la seguridad de la Nación o el interés nacional"

La aplicación generalizada del juicio oral en los procesos penales y, así como en los juicios tanto ante los tribunales de familia cuanto los del trabajo, establecida en las reformas aprobadas en todas estas materias, refuerza el principio de la publicidad de la Justicia, si bien ella puede restringirse en los procedimientos penales y de familia por resolución del tribunal dela causa.

En la misma dirección se orientan la cuenta anual que debe dar en audiencia pública el 1 demarzo de cada año el Presidentedela CorteSuprema respecto del trabajo ejecutado y del que ha quedado pendiente; la Memoria quedistribuyela Corporación A dministrativa desu gestión en el año anterior y las publicaciones y estadísticas que dan a conocer los tribunal es colegiados del os asuntos a que se refieren los artícul os 586y siguientes del Código Orgánico de Tribunales.

\section{TERRITORIALIDAD}

Los tribunales chilenos conocen y resuelven todos los asuntos quese promuevan en el territorio nacional, cual quiera sea su natural eza o la cal idad de las personas que intervengan, según lo establece el artículo $5^{\circ}$ del Código Orgánico de Tribunales, con las excepciones que fijan la Constitución Política y las leyes. 
Entre ellas, el artículo $6^{\circ}$ del mismo Código somete a la jurisdicción de los tribunales nacionales determinados asuntos criminales ocurridos fuera del territorio, por ejemplo, los cometidos por agentes diplomáticos chilenos en ejercicio de sus funciones y los ejecutados por chilenos contra chilenos, si el culpablevuelvea Chilesin haber sido juzgado, etc.

A su turno, dentro del país, los tribunales sólo deben actuar dentro del territorio que les está asignado, según su natural eza y jerarquía.

\section{INEXCUSABILIDAD}

El artículo 10 이 del Código Orgánico de Tribunales señala que, reclamada su intervención en forma legal y en negocios de su competencia, no podrán los tribunales excusar su intervención, ni aun por falta de ley que resuelva la contienda sometida a su decisión.

Esta regla se incorporó al artículo 760 dela Constitución de 1980, lo quevino a reforzar su obligatoriedad para los tribunales y a facilitar el acceso a la justicia como garantía individual específica quees elemento del régimen deEstado de Derecho a cuya existencia real colabora la acción judicial.

\section{PROCEDIMIENTOSJUDICIALES}

Los procedimientos son las vías por las cual es transita la actividad del tribunal, delas partes del juicio y deotros interesados.

En esteterreno, el sistemajudicial chileno seencuentra en un período decambios y reemplazos delos procedimientos quese han aplicado durantelargo tiempo, como consecuencia deimportantes reformas en actual implementación.

Los Códigos de Procedimiento Civil y Penal, es decir, los cuerpos que básicamente han agrupado las reglas a que deben sujetarse tanto los juicios civiles como criminales, se dictaron a comienzos del siglo XX y si bien fueron constantemente modificados, la normativa referente al proceso penal fue reemplazada por completo a contar del año $2000,{ }^{5}$ como parte del conjunto de profundas innovaciones que ha experimentado la materia.

El sistema procesal que rigió hasta esta reforma se presentaba con un carácter inquisitorio corregido y tuvo como modelo la legislación española de 1852. Se 
consolidó en el Código de Procedimiento Penal de 1906, en cuya dictación se hizo reserva expresa de que las condiciones de la comunidad chilena de aquél entonces no permitían establecer el sistema acusatorio oral.

Las características inquisitivas del procedimiento se acentuaron con la supresión en 1927 de los Promotores Fiscales, que eran acusadores públicos en primera instancia, lo que hizo que el juez pasara a asumir el triple papel de investigador, acusador público y juzgador.

Lafasedeinvestigación del delito y laindividualización del delincuente, llamada sumario, tenía carácter secreto y el afectado sólo podía defenderse, con conocimiento de los antecedentes de la investigación, después deser acusado, en la segunda etapa del proceso llamada plenario, deíndole bilateral.

En el nuevo sistema que rige actualmente en plenitud y que se llevó a cabo mediantereforma dela Carta Política, ${ }^{6}$ se radicaron las funciones de investigar los delitos y ejercer la acción pública, así como de prestar protección a las víctimas y testigos, en el $\mathrm{M}$ inisterio Público. Este es un organismo constitucionalmente autónomo, organizado por la Ley $\mathrm{N}^{\circ}$ 19.640, de 15 de octubre de 1999, distinto y ajeno al Poder Judicial, pero sujeto en cuanto a la autorización de medidas cautelares que priven al imputado o a otras personas del ejercicio de derechos asegurados por la Constitución, o quelos restrinjan o perturben, a la aprobación judicial previa a través de la intervención de los Jueces de Garantía.

Para la defensa delos imputados, secreó por la Ley N 19.718, de 10 de marzo de2001, un servicio estatal dependientedel Ministerio de Justicia, denominado D efensoría Penal Pública, encargado de prestarles asistenciasi carecen demedios para obtenerla por si solos y de licitar esta actividad entre abogados particulares.

En el nuevo procedimiento criminal, la triple función de los jueces de primer grado -investigación, acusación y juzgamiento - se transformó, por una parte, en la de cautel ar las garantías del imputado y demás afectados y, por la otra, en la de moderar la controversia entre acusación y defensa y en la de juzgadores en única instancia.

En el nuevo sistema, en que opera explícitamente la presunción de inocencia del imputado, el juzgamiento mismo del acusado se desarrolla así en una sola 
instancia, a cargo de tribunales colegiados, mediante juicios orales, públicos, concentrados e ininterrumpidos ante los jueces y con presencia de las partes. En ellos, la sentencia se comunica de inmediato, aunque se emita y lea formalmentecon posterioridad.

Los tribunales que intervienen en el proceso penal reformado, cuentan con una estructura distinta a la tradicional. Con un ComitédeJ ueces para fijar, entreotros cometidos, el procedimiento objetivo quedebeutilizarsepara la distribución de las causas entre los jueces y con una gestión operacional a cargo de un Administrador del Tribunal. De éste dependen diferentes unidades administrativas, lo que ha venido a separar las funciones propiamente jurisdiccionales de la actividad de apoyo administrativo y financiero del tribunal.

La aplicación del nuevo procedimiento penal se llevó a efecto gradual mente durante más de cinco años en distintas Regiones a través de igual número de etapas diferenciadas.

Para el Poder Judicial, importó un considerable esfuerzo en obras de construcción, remodelación, habilitación y equipamiento de los nuevos tribunales, ${ }^{7}$ así como del refuerzo de los que pasaron a ejercer funciones de juzgados de garantía, continuando con sus competencias en materias civiles, laborales o demenores. Asimismo, significó un trabajo notable en la selección, capacitación y adiestramiento, ${ }^{8}$ traslados y designaciones de jueces y funcionarios.

En ambas materias, participaron los tribunales, bajo la dirección de la Corte Suprema, la que ha dictado más de 50 A utos A cordados e Instrucciones para regular materias relacionadas con las reformas y con la debida coordinación con los restantes actores del proceso.

\section{TRIBUNALESDEFAMILIA}

Con fecha 1 deoctubre de 2005 y simultáneamenteen todo el país, iniciaron su funcionamiento los nuevos Tribunales deFami lia en reemplazo de los antiguos Juzgados de Menores, para facilitar el acceso de las personas a una justicia especializada en estos asuntos. Esta reforma agregó la mediación a la ya existente conciliación como medio no controversial de solución de conflictos, así como el refuerzo dela acción interdisci plinaria queéstos exigen, a través de 
Consejos Técnicos formados por Psicólogos y A sistentes Sociales, para proporcionar al juez asesoría profesional inmediata y permanente.

El procedimiento es oral, flexible, desformalizado, condensado y fundado en la inmediación, en términos similares al nuevo proceso penal. Su competencia es numerosa y variada en la natural eza y grado de complejidad de los asuntos.

\section{TRIBUNALES DEL TRABAJO Y DE COBRANZA PREVISIONAL}

Se encuentran publicadas y en vías de entrar en plena vigencia las leyes $\mathrm{N}^{\circ} \mathrm{S}$ 20.022 y 20.023, ambas de 31 de mayo de 2005 y 20.087, de 9 de enero de 2006, que modifican la organización judicial especializada y reemplazan el procedimiento en estas materias.

Este nuevo juicio se organiza sobre la base del modelo del proceso penal reformado, esto es, se trata deun juicio oral, concentrado y desformalizado, con reducción y reemplazo de los recursos antetribunales superiores.

\section{TRIBUNALES CIVILES}

El Ministerio de Justicia ha convocado a un foro amplio de juristas para efectuar los estudios necesarios a fin dereformar estesector dela Justicia, en consonancia con las modificaciones realizadas o que se llevan a efecto en los ámbitos indicados.

Como seindicó al comienzo, esta síntesis sólo pretendeproporcionar una visión esquemática de la organización y de los procedimientos que caracterizan al Poder Judicial chileno, en un período de implementación de las diversas reformas que se han sancionado en ambos aspectos y que se encuentran, a su turno, en distintos grados de revisión y perfeccionamiento.

\footnotetext{
Damaska, M., Las Caras de la Justicia y el Poder del Estado, Editorial Jurídica de Chile, Santiago, 2000.

Artículo $3^{\circ}$, Constitución Política de la República.

3 Santiago, la capital del país, se había fundado sólo en 1541.

4 Artículo $1^{\circ} \mathrm{N}^{\circ} 42$ de la Ley de Reforma Constitucional $\mathrm{N}^{\circ} 20.050$, de 26 de agosto de 2005 .

5 Ley $\mathrm{N}^{\circ} 19.696$, de 12 de octubre de 2000.
} 
Urbano Marín vallejo - Visión actual del sistema judicial chileno

6 Ley $\mathrm{N}^{\circ} 19.519$, de 16 de septiembre de 1997.

7 Un total de $191.037 \mathrm{~m} 2$ en nuevas obras einstalaciones.

87.447 personas a cargo de la Corporación A dministrativa. 